\title{
A FUZZY BINARY BI OBJECTIVE TRANSPORTATION MODEL: IRANIAN STEEL SUPPLY NETWORK
}

\author{
Hannan AMOOZAD MAHDIRAJI ${ }^{1^{*}}$, Moein BEHESHTI ${ }^{2}$, Seyed HosseinRAZAVI HAJIAGHA ${ }^{3}$, \\ Edmundas Kazimieras ZAVADSKAS ${ }^{4}$ \\ ${ }^{1}$ Faculty of Management, University of Tehran, Tehran, Iran \\ ${ }^{2}$ Dept of Management, University of Tehran, Kish, Iran \\ ${ }^{3}$ Dept of Management, Khatam University, Tehran, Iran \\ ${ }^{4}$ Institute of Sustainable Construction, Vilnius Gediminas Technical University, Vilnius, Lithuania
}

Received 22 August 2016; revised 28 January 2017; accepted 18 February 2017

\begin{abstract}
Prominent influence of transportation costs on supply chain overall profit indicates the importance and emergence of transportation optimization models. Regarding this issue and in view of realistic situation consisting of non-deterministic information, in this research optimizing inbound and outbound transportation costs of a multi echelon supply chain has been considered. To deal with uncertain time deliveries and pricing strategies adopted by different members of supply chain, in conjunction with unpredictable demand rate, fuzzy logic and specifically Trapezoidal Fuzzy Numbers (TrFNs) are included. After designing a fuzzy binary multi objective model based upon structural assumptions, the solving approach is proposed and the model is employed on Iranian steel supply network to illustrate the potential and advantages of our scheduled model. The bi-objective mixed integer fuzzy programming model presents and encompasses many realistic circumstances making the model applicable in network transportation cases.
\end{abstract}

Keywords: fuzzy sets, transportation problem, binary bi objective models, supply network, optimization.

\section{Introduction}

Transportation Problems (TPs) are used since the organization of human beings in to society, and today they have wide ranging applications such as scheduling, production, investment, plant location, inventory control, employment scheduling and many others (Ebrahimnejad 2014). Various models of TP are lucidly explained for the benefit of common readers (Alam, Rastogi 2011). During recent years, logistics network design has deservedly received a great deal of attention as one of the most important fields in Supply Chain Management (SCM) (Jin et al. 2011). Due to global economy downturn and competitive business environment, logistic and SCM play an important role for any firms to reduce their operations cost and enhance their competitive advantage; however, logistic cost structure is still high as a result of ineffective planning. Actually, transportation cost, the largest logistic cost component, is continuously increasing annually (Rianthong, Dumrongsiri 2012).

The solution to TP was originally developed by Hitchcock and was later advanced by Koopmans (Alam, Rastogi
2011). Their contributions motivated scholars to develop various transportation methods involving a number of shipping sources and a number of destinations (Sharma et al. 2012).

Suppose a company has $\mathrm{m}$ warehouses and $\mathrm{n}$ retail outlets. A single product is to be shipped from the warehouses to the outlets. Remark that each warehouse has a given level of supply, and each outlet has a given level of demand. Moreover, the transportation costs between every pair of warehouse and outlet is achievable, and these costs are assumed to be linear. The problem of interest is to determine an optimal transportation sketch between the warehouses and the outlets, subject to the specified supply and demand constraints or any other structural or organizational limitations. Generally, we presuppose that transportation costs, values of demand and supplies are crisp; as a matter of fact, decision makers need to consider common errors and inaccuracy of data and evidences for cost parameters, demand or supply rates (Samuel, Venkatachalapathy 2014). Fuzzy sets, stochastic programming

${ }^{*}$ Corresponding author. E-mail: h.amoozad@ut.ac.ir

This is an Open Access article distributed under the terms of the Creative Commons Attribution License (http://creativecommons.org/licenses/by/4.0/), which permits unrestricted use, distribution, and reproduction in any medium, provided the original author and source are credited. 
or grey numbers are widely implemented by researchers to discuss uncertainty circumstances of TP. Zimmermann (1978) uncovered that fuzzy linear programming method is usually efficient and accurate in uncertain circumstances.

In this paper we have considered an unlimited two echelon supply chain including suppliers from $i=\{1,2,3, \ldots, m\}$ and retailers from $j=\{1,2,3, \ldots, n\}$ supporting the transportation system of single product supply chain on the basis of three possible transportation mode. Moreover, the demand function in our proposed system is on fuzzy numbers due to market uncertainty in order to consider the possible market changes. Furthermore, this model enables optimization for a more advanced multi dominance TP to improve the price, transportation costs and time simultaneously. The main purpose of our research is modelling SC transportation system upon fuzzy demands, fuzzy time deliveries and fuzzy transportation costs; besides, using a new $0-1$ binary method to optimize transportation procedure and to minimize total transportation cost of proposed supply chain. We have developed a fuzzy mixed integer programming model based on fuzzy parameters for this purpose. Remain of the paper is organized as follows. First of all, Fuzzy Transportation Problem (FTP) considering related researches in introduced. After, preliminaries of fuzzy sets and theory is illustrated; subsequently, our fuzzy binary bi objective TP regarding structure, assumptions, model and solving approach is illustrated. Finally a case study related to Iranian steel industry and related supply network is presented and the proposed model is applied.

\section{Fuzzy transportation problem}

Since the identification of the TP in 1941 and its efficient solution by the simplex method in 1947 , several researchers have developed models and algorithms in different situations having different variables; being similar in structure to the models and algorithms of the standard TP (Alam, Rastogi 2011). TP is one of the fundamental problems of network flow usually used to minimize the transportation cost for industries with numerous resources and destinations while satisfying the supply limit and demand requirement (Sharma et al. 2012). Classical TP aims to minimize the total cost for shipping various capacities of commodities on the requirement of destinations from the available sources. TP model often can be built as a linear programming model or an NP-hard problem.

Generally the transportation cost of one unit of a commodity is depending on the source and the destination (Purusotham, Murthy 2012). Many practical logistics problems may be more complex than the general model (Stević et al. 2017; Petraška et al. 2017, etc.). As an extended model, multi-product TP selects the best route considering several kinds of products (Jin et al. 2011).

The general TP can be solved by the simplex method; nevertheless, simpler method for generating optimal solutions for bi-criteria TP from which the best transportation plan can be selected by the decision maker is previously figured (Alam, Rastogi 2011).

Considering uncertainty in TPs modeling is a fact that scholars need to consider in their researches. Dealing with uncertain circumstances are categorized in 3 clusters: (1) Statistic and probability; (2) Fuzzy sets theory; (3) Grey system (Liu, Lin 2006; Amoozad Mahdiraji et al. 2016). Deng (1989) introduced Grey theory and there's been a massive amount of researches on this theory. i.e. Amoozad Mahdiraji et al. (2011, 2016), Razavi Hajiagha et al. (2014). Although the advantage of this theory is that it presupposes the probability distribution or membership function form of information (Li et al. 2014; Amoozad Mahdiraji et al. 2016) and scholars used this method to solve TP (Bai et al. 2004); nonetheless, we propose a new fuzzy binary approach to optimize supply chain considering time and cost as two treasure resources that every manager needs to minimize. Fuzzy logic and fuzzy numbers are the main tools performing for this purpose.

Chanas et al. (1984) worked on TP considering supplies and demands being fuzzy and solved them via parametric programming method, TP has also been investigated conditions that cost coefficients were assumed to be fuzzy. Chanas and Kuchta (1996) solved this case upon crisp objective function after its conversion to a bi criterial function. Supplies, demands and conveyance capacities were presupposed in Trapezoidal Fuzzy Number (TrFN) sets and TP got solved in a parametric approach (Jiménez, Verdegay 1998, 1999). Optimal solution of TP with fuzzy demand and fuzzy product was identified by Chiang (2005). Furthermore, some new approaches for solving FTPs were proposed (Basirzadeh 2011; Kumar et al. 2010; Güzel 2010). Lin (2009) employed a genetic algorithm to solve TP with fuzzy demand and supply coefficients and ranking fuzzy numbers with signed-distance measurement were applied for the evaluation and selection of the algorithm. Pandian and Natarajan (2010) denoted a new algorithm namely, fuzzy zero point method for finding fuzzy optimal solution for such FTP in which the transportation cost, supply and demand are represented by TrFNs.

In 2012 mathematical models for optimal production, inventory and transportation planning with direct shipment was proposed (Poonam et al. 2012; Mohanaselvi, Ganesan 2012; Ritha, Vinotha 2009). Similarly, some revised simplex methods on fuzzy logic and fuzzy numbers were proposed for solving TP (Kumar, Murugesan 2012; Gani et al. 2011). Jin et al. (2011) presented a multi-product two-stage TP model with multi-time period and inventory using priority-based genetic algorithm. Scholars have also used type-2 fuzzy parameters in the resent years (Kundu et al. 2015; Liu et al. 2014; Pramanik et al. 2015). Kocken and Sivri (2016) set fuzzy cost coefficients, fuzzy supplies, fuzzy demands and fuzzy conveyances to develop a parametric method to generate all optimal solutions of fuzzy solid TP.

Considering the purpose of our research, which targets modelling unlimited two echelon supply chain TP with 
multi products, Purusotham and Murthy (2012) proposed a model with NP-Hard nature problem, where several commodities are produced in several plant sites with capacity constraints, and distributed to several destination sites according to demands and transportation constraints. Predominantly, in the vast majority of cases previous researches were focused on a single object problem, deterministic demand rate, and deterministic parameters in transportation besides limited uncertain information for time, price or demand. However, in the proposed model, multi objective problem, fuzzy demand rate, fuzzy time and prices besides vessel transport selection possibility are considered; eventually, the complex fuzzy binary bi objective model is solved by a novel procedure. Next section of this paper is a brief review of some preliminaries of fuzzy set theory.

\section{Preliminaries of fuzzy set theory}

The concept of fuzzy sets was introduced by Zadeh (1965) and it has been used ever since to solve many practical problems. An assumed fuzzy number like $A$ is delineated as an interval $\left[a_{l}, a_{u}\right]$. It is conspicuous that $a_{l}$ is the lower boundary of $A$ and $a_{u}$, the upper. Respectively $\bar{a}_{\alpha}^{L}$ and $\bar{a}_{\alpha}^{U}$ are defined as (Wang 2015):

$$
\begin{aligned}
& \bar{a}_{\alpha}^{L}=\inf (z)_{\mu_{\bar{a}}(z) \geq \alpha} ; \\
& \bar{a}_{\alpha}^{U}=\sup (z)_{\mu_{\bar{a}}(z) \geq \alpha} ;
\end{aligned}
$$

$F(\mathbb{R})$ is the set of fuzzy numbers for any $\alpha \in(0,1]$ and $\left[\bar{a}_{\alpha}^{L}(0), \bar{a}_{\alpha}^{U}(0)\right]=a_{0}$.

We define expected interval $E I(\bar{a})$ and expected value $E V(\bar{a})$ for a fuzzy number like $\bar{a}$ as follow (Heilpern 1992; Dubois, Prade 1987):

$$
\begin{aligned}
& E I(\bar{a})=\left[E_{\star}(\bar{a}), E^{\star}(\bar{a})\right]=\left[\int_{0}^{1} \bar{a}_{\alpha}^{L}(\beta) d \beta, \int_{0}^{1} \bar{a}_{\alpha}^{U}(\beta) d \beta\right] ; \\
& E V(\bar{a})=\frac{1}{2}\left(E_{\star}(\bar{a})+E^{*}(\bar{a})\right) .
\end{aligned}
$$

Suppose $\bar{a}$ as a fuzzy number (Chanas 2001; Delgado et al. 1998; Grzegorzewski 1998, 2008) have discussed ambiguity $\operatorname{Amb}(\bar{a})$, value $\operatorname{Val}(\bar{a})$, width $w(\bar{a})$, left-hand ambiguity $A m b_{L}(\bar{a})$, right-hand ambiguity $A m b_{U}(\bar{a})$ as below:

$$
\begin{aligned}
& A m b(\bar{a})=\int_{0}^{1} \beta \cdot\left(\bar{a}_{\alpha}^{L}(\beta)-\bar{a}_{\alpha}^{U}(\beta)\right) d \beta \\
& \operatorname{val}(\bar{a})=\int_{0}^{1} \beta \cdot\left(\bar{a}_{\alpha}^{L}(\beta)+\bar{a}_{\alpha}^{U}(\beta)\right) d \beta \\
& w(\bar{a})=\int_{0}^{1}\left(\bar{a}_{\alpha}^{L}(\beta)-\bar{a}_{\alpha}^{U}(\beta)\right) d \beta ; \\
& A m b_{L}(\bar{a})=\int_{0}^{1} \beta \cdot\left(E V(\bar{a})-\bar{a}_{\alpha}^{L}(\beta)\right) d \beta ; \\
& \operatorname{Amb}_{U}(\bar{a})=\int_{0}^{1} \beta \cdot\left(\bar{a}_{\alpha}^{U}(\beta)-E V(\bar{a})\right) d \beta .
\end{aligned}
$$

Euclidean distance is one of the most illustrious metrics defined by Grzegorzewski (1998):

$$
\begin{aligned}
& d^{2}(\bar{a}, \bar{b})=\int_{0}^{1}\left(\bar{a}_{\alpha}^{L}(\beta)-\bar{b}_{\alpha}^{L}(\beta)\right)^{2} d \beta+ \\
& \int_{0}^{1}\left(\bar{a}_{\alpha}^{U}(\beta)-\bar{b}_{\alpha}^{U}(\beta)\right)^{2} d \beta .
\end{aligned}
$$

Scholars usually discuss fuzzy numbers as triangular and trapezoidal, a schematic view of triangular and trapezoidal numbers is given in Figure 1. We illustrate the main operations and basic concepts of trapezoidal numbers in the rest of this section.

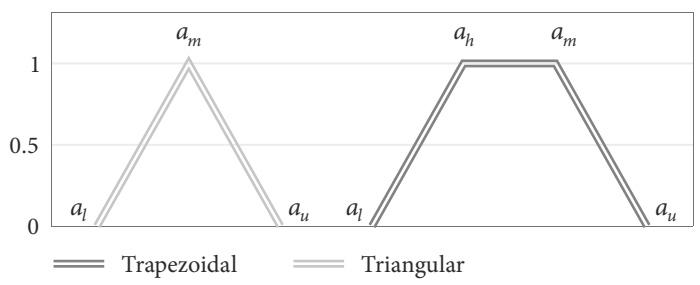

Figure 1. Schematic view of triangular and trapezoidal

Trapezoidal numbers are more accurate and comprises the triangular numbers, therefore scholars use this method more widely (Ban, Coroianu 2014). A TrFN $\bar{a}, \bar{a}_{\beta}=\left[\bar{a}_{L}(\beta), \bar{a}_{U}(\beta)\right], \beta \in[0.1]$, is given by:

$$
\bar{a}_{L}(\beta)=a_{l}+\left(a_{h}-a_{l}\right) \cdot \beta
$$

and

$$
\bar{a}_{U}(\beta)=a_{u}+\left(a_{u}-a_{m}\right) \cdot \beta .
$$

Note that if $a_{l} \leq a_{h} \leq a_{m} \leq a_{u}, \quad \bar{a}$ would be: $\bar{a}=\left(a_{l}, a_{h}, a_{m}, a_{u}\right)$. The above form is sometimes represented as Yeh (2008) $\bar{a}_{L}(\beta)=a_{l}+a_{m} \cdot\left(\beta-\frac{1}{2}\right)$, $\bar{a}_{U}(\beta)=a_{a_{h}}-a_{u} \cdot\left(\beta-\frac{1}{2}\right)$, where $a_{l}, a_{h}, a_{m}, a_{u} \in \mathbb{R}$. The membership function $\left(\mu_{\bar{A}}\right)$ of a TrFN like $A$, which can be denoted as a quartet $\left(a_{l}, a_{h}, a_{m}, a_{u}\right)$ is as below (Chen, S.-J., Chen, S.-M. 2007; Kumar, Gupta 2011):

$$
\mu_{\bar{A}}(x)=\left\{\begin{array}{ll}
\frac{x-a_{l}}{a_{h}-a_{l}}, & a_{l} \leq x \leq a_{h} ; \\
1, & a_{h} \leq x \leq a_{m} ; \\
\frac{a_{u}-x}{a_{u}-a_{m}}, & a_{m} \leq x \leq a_{u} ; \\
0, & \text { otherwise. }
\end{array}\right\}
$$

Considering $\bar{a}=\left(a_{l}, a_{h}, a_{m}, a_{u}\right)$ and $\bar{b}=\left(b_{l}, b_{h}, b_{m}, b_{u}\right)$ as two TrFN, the arithmetic operations on these two numbers is designated as below (Chen, S.-J., Chen, S.-M. 2007; Kumar, Gupta 2011):

$$
\begin{aligned}
& \lambda \geq 0, \lambda \cdot \bar{a}=\left(\lambda \cdot a_{l}, \lambda \cdot a_{h}, \lambda \cdot a_{m}, \lambda \cdot a_{u}\right) ; \\
& \lambda \leq 0, \lambda \cdot \bar{a}=\left(\lambda \cdot a_{u}, \lambda \cdot a_{m}, \lambda \cdot a_{h}, \lambda \cdot a_{l}\right) ; \\
& \bar{a}+\bar{b}=\left(a_{l}+b_{l}, a_{h}+b_{h}, a_{m}+b_{m}, a_{u}+b_{u}\right) ; \\
& \bar{a}-\bar{b}=\left(a_{l}-b_{u}, a_{h}-b_{m}, a_{m}-b_{h}, a_{u}-b_{l}\right) .
\end{aligned}
$$


Generally speaking, if we assume "o" as an operation on real numbers $(+,-, \wedge, \vee, \ldots)$ between $a$ and $b$, we can define an extended operation on fuzzy sets (suppose $\bar{a}$, $\bar{b}$ as ${ }^{*}$ two fuzzy numbers) (Wang 2015):

$$
\begin{aligned}
& \mu_{\bar{a} \circ \bar{b}}(z)=\sup \left\{\mu_{\bar{a}}(x) \wedge \mu_{\bar{b}}(y)\right\} ; \\
& x, y: z=x \circ y .
\end{aligned}
$$

If we assume $\bar{a}=\left(a_{l}, a_{h}, a_{m}, a_{u}\right)$ and $\bar{b}=\left(b_{l}, b_{h}, b_{m}, b_{u}\right)$ as two $\operatorname{TrFN}$, then their Euclidean distance mentioned in Equation (10) would be:

$$
\begin{aligned}
& d^{2}(\bar{a}, \bar{b})=\left(a_{l}-b_{l}\right)^{2}+\left(a_{u}-b_{u}\right)^{2}+ \\
& \frac{1}{12} \cdot\left(a_{m}-b_{m}\right)^{2}+\frac{1}{12} \cdot\left(a_{h}-b_{h}\right)^{2} .
\end{aligned}
$$

The extended trapezoidal approximation is used to minimize the distance of $d(P, Q)$, where $Q \in F_{e}^{\bar{\alpha}}(\mathbb{R})$, although (Allahviranloo, Firozja 2007) believes that $\bar{a}_{e}(P)=\left[a_{l e}(P), a_{\text {he }}(P), a_{m e}(P), a_{u e}(P)\right]$ as the extended trapezoidal approximation is not always a fuzzy number, it is determined it as below:

$$
\begin{aligned}
& a_{l e}(P)=\int_{0}^{1} \bar{a}_{\alpha}^{L}(\beta) d \beta \\
& a_{h e}(P)=\int_{0}^{1} \bar{a}_{\alpha}^{U}(\beta) d \beta ; \\
& a_{m e}(P)=\frac{1}{12} \cdot \int_{0}^{1}\left(\beta-\frac{1}{2}\right) \cdot \bar{a}_{\alpha}^{L}(\beta) d \beta ; \\
& a_{u e}(P)=-\frac{1}{12} \cdot \int_{0}^{1}\left(\beta-\frac{1}{2}\right) \cdot \bar{a}_{\alpha}^{U}(\beta) d \beta,
\end{aligned}
$$

where:

$$
a_{m e}(P) \geq 0 \text { and } a_{u e}(P) \geq 0 .
$$

If we define $F(\mathbb{R})$ as a set of fuzzy numbers on set of real numbers, then upon a ranking function proposed by Kaur and Kumar (2012) the ranking function is $\mathfrak{R}: F(\mathbb{R}) \rightarrow \mathbb{R}$ and in case of comparison between two fuzzy numbers like $\bar{a}=\left(a_{l}, a_{h}, a_{m}, a_{u}\right)$ and $\bar{b}=\left(b_{l}, b_{h}, b_{m}, b_{u}\right)$ the aforementioned function maps them into the real line. i.e. (Ebrahimnejad 2014):

$\bar{a} \tilde{\leq} \bar{b}$, if and only if

$$
\begin{aligned}
& \mathfrak{R}(\bar{a})=\frac{a_{l}+a_{h}+a_{m}+a_{u}}{4} \leq \mathfrak{R}(\bar{b})= \\
& \frac{b_{l}+b_{h}+b_{m}+b_{u}}{4}
\end{aligned}
$$

$\bar{a} \tilde{\geq} \bar{b}$, if and only if

$$
\begin{aligned}
& \mathfrak{R}(\bar{a})=\frac{a_{l}+a_{h}+a_{m}+a_{u}}{4} \leq \mathfrak{R}(\bar{b})= \\
& \frac{b_{l}+b_{h}+b_{m}+b_{u}}{4}
\end{aligned}
$$

$\bar{a} \cong \bar{b}$, if and only if

$$
\begin{aligned}
& \mathfrak{R}(\bar{a})=\frac{a_{l}+a_{h}+a_{m}+a_{u}}{4}=\mathfrak{R}(\bar{b})= \\
& \frac{b_{l}+b_{h}+b_{m}+b_{u}}{4} .
\end{aligned}
$$

\section{Proposed binary multi objective fuzzy transportation model}

\subsection{Model structure and assumptions}

Consider a two echelon supply chain encompassing unlimited suppliers (sellers) and retailers (buyers) catering a single product. As previously mentioned the main target of this research is to minimize the total transportation costs of the informed supply chain besides identifying the transportation vessel; therefore, the overall profit increases and all members will be satisfied. Transportation vessels include rail (train), truck and sea transportation systems differing for each supplier upon routs capabilities and infrastructures. Two main objectives of our proposed model are to identify the transportation mode; furthermore, determine the quantity transported from any supplier to each retailer. The initial scheme of the elaborated SC is demonstrated in Figure 2.

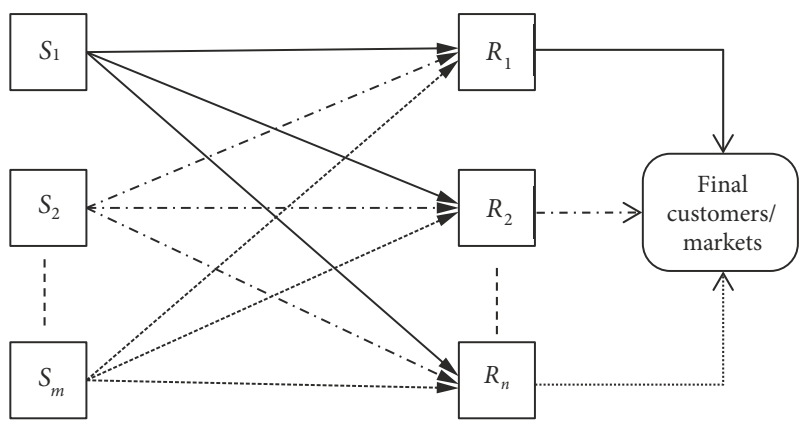

Figure 2. Considered supply network

Moreover, other assumptions regarding the illustrated supply chain are considered as fellow:

1) The above supply chain includes $m$ suppliers indexed by $S_{i}$ while $i=\{1,2, \ldots, m\}$ and $n$ retailers indexed by $R_{j}$ while $j=\{1,2, \ldots, n\}$.

2) Demand function depends on the price offered by each retailer to final customer or market, being a nonlinear function equal to $D_{j}=K_{j} \cdot C_{P_{j}}^{-\alpha_{j}}$ where alpha indicates the inverse effect of price for each retailer, (Esmaeili et al. 2008; Jia et al. 2013, Amoozad Mahdiraji et al. 2015), $K_{j}$ stands for price fixed coefficient $C_{P j}$ illustrates the price offered by each retailer to the market or final customer and $D_{j}$ presents the demand related to $j$-th retailer. One of the novelties of our research is considering uncertainty and nondeterministic situations for transportation in supply chain. With this fact in mind, the offered price of each retailer is considered as TrFNs and presented as $\bar{C}_{P_{j}}$; accordingly, the demand function transfers to $\bar{D}_{j}=K_{j} \cdot \bar{C}_{P_{j}}^{-\alpha_{j}}$.

3) Each supplier on the basis of transportation infrastructures and possible routes is capable to transfer the requested demand by rail, truck and sea transportation system, identified by $L$ while $L=1$ presents sea, $L=2$ presents truck and $L=3$ presents rail transportation system. 
4) By identifying the transportation mode, besides determining the transportation quantity, two objective functions are considered. The first aims to select the transportation mode considering the lowest delivery time and the second object targets minimizing purchase and transportation costs (inbound and outbound transportations).

5) Transportation costs and delivery time may vary subjected to different circumstances; hence, their amounts are not deterministic and accurate. Fuel prices, political and economic issues, weather changes and many other determinant factors may engender changes in costs and time. With this fact in mind and to increase the novelty and complexity of our research, transportation costs and delivery time are considered uncertain and presented as TrFNs in our proposed model. Remark that $\bar{t} \& \bar{p}$ present time and transportation costs upon TrFNs. Other decision variables and parameters related to the figured model are denoted in Table 1.

Regarding the structure and models assumptions, the initial sketch of the network manner is indicated in Figure 3.

\subsection{Modelling process}

The proposed model upon the aforementioned structure and assumptions and similar to any mathematical multi objective model, consists of a bi objective function subjected to structural limitations. As previously mentioned, two main objectives including identifying the transportation mode, besides determining the transportation quantity are studied, where the first $\left(Z_{1}\right)$ aims to select

$$
S_{i} \stackrel{\bar{t}_{i j l} ; \bar{p}_{i j l} ; x_{i j l}}{\longrightarrow} R_{j} \stackrel{\bar{D}_{j}=k_{j} \bar{C}_{P_{j}}^{-\alpha_{j}}}{\longrightarrow} \text { Final Customers/Markets }
$$

Figure 3. Initial manner of the supply network

the transportation mode regarding the delivery time and the second object $\left(Z_{2}\right)$ targets minimizing purchase and transportation costs; therefore, Equation (27) eventuates.

$$
\begin{aligned}
& \operatorname{Min} Z_{1}=\sum_{i=1}^{m} \sum_{j=1}^{n} \sum_{l=1}^{e} \bar{P}_{i j l} \cdot X_{i j l} ; \\
& \operatorname{Min} Z_{2}=\sum_{i=1}^{m} \sum_{j=1}^{n} \sum_{l=1}^{e} \bar{T}_{i j l} \cdot Y_{i j l} .
\end{aligned}
$$

The constraint of our proposed model embraces two parts. First, the classical transportation limitations consisting of demand fulfilment and supply utilization; moreover, some minimum requirements and maximum capacities are definable for any transportation mode. Demand fulfilment for each retailer is figured as Equation (28), while supply utilization of each supplier capacity is illustrated in Equation (29):

$$
\begin{aligned}
& \sum_{i=1}^{m} \sum_{l=1}^{e} X_{i j l}=\bar{D}_{j}, \quad \forall j \in n ; \\
& \sum_{j=1}^{n} \sum_{l=1}^{e} X_{i j l} \leq C a_{i} .
\end{aligned}
$$

\begin{tabular}{|c|c|c|c|c|}
\hline Type & Amount & Symbol & Definition & Range \\
\hline \multirow{8}{*}{ Parameter } & \multirow{8}{*}{ Crisp } & $S_{i}$ & The $i$-th supplier & $i=\{1,2, \ldots, m\}$ \\
\hline & & $R_{j}$ & The $j$-th retailer & $j=\{1,2, \ldots, n\}$ \\
\hline & & $L$ & The transportation mode & $l=\{1,2, \ldots, e\}$ \\
\hline & & $K_{j}$ & Demand coefficient for $j$-th retailer & $K>0$ \\
\hline & & $\alpha_{j}$ & Price demand coefficient for $j$-th retailer & $\alpha>1$ \\
\hline & & $C a_{i}$ & The possible capacity of $i$-th supplier & - \\
\hline & & $m_{i j l}$ & Minimum order for employing $l$-th vessel & - \\
\hline & & $u_{i j l}$ & Maximum capacity possible for $l$-th vessel & - \\
\hline Function & \multirow{4}{*}{ TrFNs } & $\bar{D}_{j}$ & Market demand for $j$-th retailer & - \\
\hline \multirow{3}{*}{ Parameter } & & $\bar{C}_{P_{j}}$ & Selling price for $j$-th retailer & - \\
\hline & & $\bar{p}_{i j l}$ & $\begin{array}{l}\text { The price and transportation costs of goods from } i \text {-th supplier } \\
\text { to } j \text {-th retailer by lth vessel }\end{array}$ & $\bar{p}_{i j l} \geq 0$ \\
\hline & & $\bar{t}_{i j l}$ & $\begin{array}{l}\text { The transfer time of transported goods from } i \text {-th supplier } \\
\text { to } j \text {-th retailer by } l \text {-th vessel }\end{array}$ & $\bar{t}_{i j l} \geq 0$ \\
\hline \multirow{2}{*}{$\begin{array}{l}\text { Decision } \\
\text { variable }\end{array}$} & \multirow[b]{2}{*}{ Variable } & $x_{i j l}$ & $\begin{array}{l}\text { The quantity of transported goods from } i \text {-th supplier } \\
\text { to } j \text {-th retailer by } l \text {-th vessel }\end{array}$ & $x_{i j l} \geq 0$ \\
\hline & & $y_{i j l}$ & $\begin{array}{l}\text { The } l \text {-th vessel used or not employed to transport goods } \\
\text { from } i \text {-th supplier to } j \text {-th retailer }\end{array}$ & $y_{i j l}\left\{\begin{array}{l}0 \\
1\end{array}\right.$ \\
\hline
\end{tabular}

Employing each transportation system depends on the order quantity; thus, least $\left(m_{i j l}\right)$ and utmost $\left(u_{i j l}\right)$ order

Table 1. Model parameters and variables 
size are considered. Larger replenishment requires transportation systems with higher capacity such as sea or rail; on the other hand, truck transportation system is more applicable for lower bath sizes. In addition, some orders may be transferred by multiple systems from supplier to retailer. The above considerations are formulated as below; where, Equation (30) indicates the minimum capacity for employing any vessel and Equation (31) presents the highest possible capacity for any transportation system:

$$
\begin{aligned}
& X_{i j l} \geq m_{i j l} \cdot Y_{i j l}, \forall_{i \in m, l \in e} ; \\
& X_{i j l} \leq u_{i j l} \cdot Y_{i j l}, \forall_{i \in m, l \in e} .
\end{aligned}
$$

Considering structure, assumptions, objective functions, constraints, decision variables and parameters, our final proposed model is demonstrated as below:

$$
\begin{aligned}
& \operatorname{Min} Z_{1}=\sum_{i=1}^{m} \sum_{j=1}^{n} \sum_{l=1}^{e} \bar{P}_{i j l} \cdot X_{i j l} ; \\
& \operatorname{Min} Z_{2}=\sum_{i=1}^{m} \sum_{j=1}^{n} \sum_{l=1}^{e} \bar{T}_{i j l} \cdot Y_{i j l}
\end{aligned}
$$

subject to

$$
\begin{aligned}
& \sum_{i=1}^{m} \sum_{l=1}^{e} X_{i j l}=\bar{D}_{j}, \forall_{j \in n} ; \\
& \sum_{j=1}^{n} \sum_{l=1}^{e} X_{i j l} \leq C a_{i}, \forall_{i \in m} ; \\
& X_{i j l} \geq m_{i j l} \cdot Y_{i j l}, \quad \forall_{i \in m, l \in e} ; \\
& X_{i j l} \leq u_{i j l} \cdot Y_{i j l}, \forall_{i \in m, l \in e} ; \\
& \bar{D}_{j}=K_{j} \cdot \bar{C}_{P_{j}}^{-\alpha_{j}}, \forall_{j \in n} ; \\
& y_{i j l}: 0 \text { or } 1 ; \\
& x_{i j l} \geq 0 ; \\
& i=\{1,2, \ldots, m\} ; \\
& j=\{1,2, \ldots, n\} ; \\
& l=\{1,2, \ldots, e\} .
\end{aligned}
$$

\subsection{Model solving approach}

The problem in Equation (31) is a bi-objective mixed integer fuzzy programming problem. The extended form of this problem can be shown as:

$$
\begin{aligned}
& \operatorname{Min} Z_{1}=\sum_{i=1}^{m} \sum_{j=1}^{n} \sum_{l=1}^{e}\left(P_{i j l}^{l}, P_{i j l}^{h}, P_{i j l}^{m}, P_{i j l}^{u}\right) \cdot X_{i j l} ; \\
& \operatorname{Min} Z_{2}=\sum_{i=1}^{m} \sum_{j=1}^{n} \sum_{l=1}^{e}\left(T_{i j l}^{l}, T_{i j l}^{h}, T_{i j l}^{m}, T_{i j l}^{u}\right) \cdot Y_{i j l} \\
& \text { subject to } \\
& \sum_{i=1}^{m} \sum_{l=1}^{e} X_{i j l}=k_{j}\left(\left(C_{p_{j}}^{l}\right)^{\alpha_{j}},\left(C_{p_{j}}^{h}\right)^{\alpha_{j}},\right. \\
& \left.\left(C_{p_{j}}^{m}\right)^{\alpha_{j}},\left(C_{p_{j}}^{u}\right)^{\alpha_{j}}\right), \forall \forall_{j \in n} ; \\
& \sum_{j=1}^{n} \sum_{l=1}^{e} X_{i j l} \leq C a_{i}, \forall_{i \in m} ;
\end{aligned}
$$

$$
\begin{aligned}
& X_{i j l} \geq m_{i j l} \cdot Y_{i j l}, \quad \forall_{i \in m, l \in e} ; \\
& X_{i j l} \leq u_{i j l} \cdot Y_{i j l}, \quad \forall_{i \in m, l \in e} ; \\
& \bar{D}_{j}=K_{j} \cdot \bar{C}_{P_{j}}^{-\alpha_{j}}, \forall_{j \in n} ; \\
& y_{i j l}: 0 \text { or } 1 ; \\
& x_{i j l} \geq 0 ; \\
& i=\{1,2, \ldots, m\} ; \\
& j=\{1,2, \ldots, n\} ; \\
& l=\{1,2, \ldots, e\} .
\end{aligned}
$$

To solve this problem, first consider the constraints set. Among the constraints, just the first one is a fuzzy equality. According to Jiménez and Verdegay $(1998,1999)$, the fuzzy equality constraint is handled using the concept of expected intervals. Jiménez and Verdegay (1998) defined the degree of among two fuzzy numbers $\bar{a}$ and $\bar{b}$ as follows:

$$
\mu_{M}(\bar{a}, \bar{b})= \begin{cases}0, & \text { if } 0 \in E_{2}^{a}-E_{1}^{b} \prec 0 ; \\ E, & \text { if } 0 \in\left[E_{1}^{a}-E_{2}^{b}, E_{2}^{a}-E_{1}^{b}\right], \\ 1, & \text { if } 0 \in E_{2}^{a}-E_{1}^{b} \prec 0,\end{cases}
$$

where:

$$
E=\frac{E_{2}^{a}-E_{1}^{b}}{E_{2}^{a}-E_{1}^{a}+E_{2}^{b}-E_{1}^{b}} .
$$

If $\mu_{M}(\bar{a}, \bar{b}) \geq \alpha$, then $\bar{a}$ is greater than or equal to $\bar{b}$ at least at the level of $\alpha$. Considering a constraint of the type $\bar{a} \cdot x \leq \bar{b}$, this constraint can be transformed into $\mu_{M}(\bar{a} \cdot x, \bar{b}) \leq \alpha$. In a satisfaction degree of $\alpha$, this inequality is transformed into:

$$
\begin{aligned}
& \left(\alpha \cdot\left(a_{l}+a_{h}\right)+(1-\alpha) \cdot\left(a_{m}+a_{u}\right)\right) \cdot x \leq \\
& \alpha \cdot\left(a_{m}+a_{u}\right)+(1-\alpha) \cdot\left(a_{l}+a_{h}\right) .
\end{aligned}
$$

For equality type constraint, "=" sign is substituted with/by " $\leq$ " in Equation (40). Therefore, the fuzzy constraints of the problem in Equation (31) are substituted by the following relation:

$$
\begin{aligned}
& \sum_{i=1}^{m} \sum_{l=1}^{e} X_{i j l}=\alpha \cdot k_{j}\left[\left(C_{p_{j}}^{m}\right)^{\alpha_{j}}+\left(C_{p_{j}}^{u}\right)^{\alpha_{j}}\right]+ \\
& (1-\alpha) \cdot k_{j}\left[\left(C_{p_{j}}^{l}\right)^{\alpha_{j}}+\left(C_{p_{j}}^{h}\right)^{\alpha_{j}}\right] ; \forall_{j \in n} .
\end{aligned}
$$

Substituting this relation in the constraints set, the fuzzy constraints are linearized. Suppose that for ease of notation, the set of constraints is shown as $x \in X$.

Considering objective functions, it is clear that an ideal transportation plan is a plan, which meets demands with lowest possible cost and time. Therefore, the ideal time $T^{+}$ and cost $C^{+}$can be found by solving the below problems, respectively:

$$
\begin{aligned}
& \operatorname{Min} E V\left(Z_{1}\right) \\
& \text { subject to } \\
& x \in X
\end{aligned}
$$


and for time criterion:

$$
\begin{aligned}
& \operatorname{Min} E V\left(Z_{2}\right) \\
& \text { subject to } \\
& x \in X,
\end{aligned}
$$

where:

$$
E V\left(Z_{1}\right)=\sum_{i=1}^{m} \sum_{j=1}^{n} \sum_{l=1}^{e} \frac{1}{4} \cdot\left(P_{i j l}^{l}+P_{i j l}^{h}+\underset{i j l}{m}+\underset{i j l}{u}\right) \cdot X_{i j l}
$$

and

$$
E V\left(Z_{2}\right)=\sum_{i=1}^{m} \sum_{j=1}^{n} \sum_{l=1}^{e} \frac{1}{4} \cdot\left(T_{i j l}^{l}+T_{i j l}^{h}+T_{i j l}^{m}+T_{i j l}^{u}\right) \cdot Y_{i j l} \cdot
$$

On the other hand, the nadir-ideal solutions for cost $C^{-}$and time $T^{-}$can be obtained by solving problems in Equations (37) and (38), by changing the optimization direction from "min" to "max".

Now, to find the satisficing solution of the problem in Equation (33) can be found by solving the below problem in a satisfaction level of $\alpha$ :

$$
\operatorname{Min} \frac{C^{-}-E V\left(Z_{1}\right)}{C^{-}-C^{+}}+\frac{T^{-}-E V\left(Z_{2}\right)}{T^{-}-T^{+}}
$$

subject to

$x \in X$;

$C^{+} \leq E V\left(Z_{1}\right) \leq C^{-}$;

$T^{+} \leq E V\left(Z_{2}\right) \leq T^{-}$.

The above procedure can be summarized as follows:

- Step 0. Gather the data required to construct the model;

- Step 1. Construct the problem according to Equation (33);

- Step 2. Transform the fuzzy constraints into nonfuzzy constraints using Equation (36);

- Step 3. Determine the ideal and nadir-ideal solutions for cost and time by solving the problems in Equations (37) and (38) respectively;

- Step 4. Set $\alpha=\{0,0.1,0.2, \ldots, 1\}$;

- Step 5. Formulate and solve the problem in Equation (40) for different values of $\alpha$;

- Step 6. Illustrate the obtained solutions for different values of $\alpha$ to decision maker(s) and select the appropriate one as the final solution.

It is notable that the above binary problems can be solved easily using optimization packages like Lingo.

\section{Iranian steel supply network}

To shed more light on elaborated model and to prove and verify the applicability of our proposed model, the fuzzy binary bi objective transportation model is employed in Iranian steel industry network. The steel industry main buyers or retailers in Iran with nearly $80 \%$ market share are Z.A $\left(R_{1}\right)$ and F.M $\left(R_{2}\right)$ since 1980 , using iron ore as their main input for manufacturing system. Iron ore is catered from domestic or foreign suppliers. The only domestic mining system being capable to meet the buyers demand is G.G $\left(S_{1}\right)$; nonetheless, in the vast majority of cases the remained required iron ore is imported from mines in Russia $\left(S_{2}\right)$, China $\left(S_{3}\right)$ and Kazakhstan $\left(S_{4}\right)$. Figure 4 illustrates the supply network including possible transportation modes. Reducing transportation costs and the selection of transportation mode are retailers and steel supply network controversial issues.

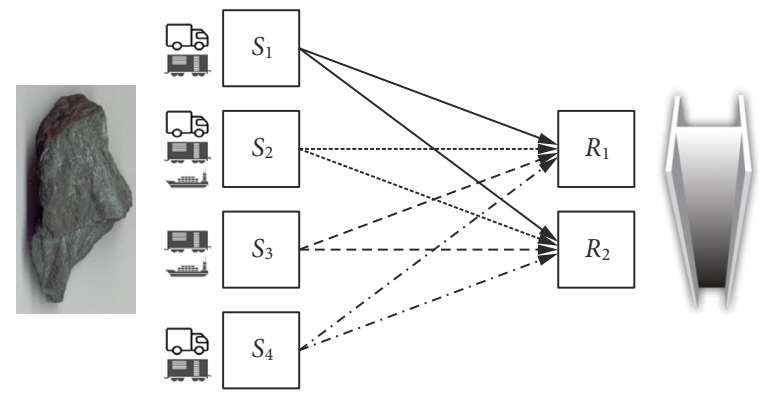

Figure 4. Iranian steel industry network

Accordingly, the steel industry supply network encompasses 4 suppliers and 2 retailers or buyers. It is noticeable that all three transportation modes are possible for the second; however, the first and second suppliers are handling truck and train; furthermore, the third supplier is capable of truck and rail transportation systems. Deterministic parameters related to aforementioned network are presented in Table 2 .

Moreover, uncertain quantities compromising selling

\begin{tabular}{|c|c|c|}
\hline Definition & Symbol & Quantity \\
\hline \multirow{2}{*}{$\begin{array}{l}\text { Demand coefficient for } \\
j \text {-th retailer }\end{array}$} & $K_{1}$ & $1.4 \cdot 10^{8}$ \\
\hline & $K_{2}$ & $1.2 \cdot 10^{8}$ \\
\hline \multirow{2}{*}{$\begin{array}{l}\text { Price demand coefficient for } \\
j \text {-th retailer }\end{array}$} & $\alpha_{1}$ & 1.1 \\
\hline & $\alpha_{2}$ & 1.3 \\
\hline \multirow{4}{*}{$\begin{array}{l}\text { The production capacity of } \\
i \text {-th supplier }\end{array}$} & $C a_{1}$ & 5 million ton \\
\hline & $C a_{2}$ & 50 million ton \\
\hline & $\mathrm{Ca}_{3}$ & 50 million ton \\
\hline & $\mathrm{Ca}_{4}$ & 20 million ton \\
\hline \multirow{3}{*}{$\begin{array}{l}\text { Minimum order for employing } \\
l \text {-th vessel }\end{array}$} & $m_{i j 1}$ & 15000 ton \\
\hline & $m_{i j 2}$ & 1500 ton \\
\hline & $m_{i j 3}$ & 5000 ton \\
\hline \multirow{3}{*}{$\begin{array}{l}\text { Maximum capacity possible for } \\
l \text {-th vessel }\end{array}$} & $u_{i j 1}$ & 25 million ton \\
\hline & $u_{i j 2}$ & 12 million ton \\
\hline & $u_{i j 3}$ & 15 million ton \\
\hline
\end{tabular}
price, transportation costs and transferring time are delineated in Tables 3, 4 and 5 upon TrFNs emanated from managerial brain storming sessions and calculations, on the basis of different scenarios.

Table 2. Parameters of steel supply network

Table 3. Selling price for each retailer

\begin{tabular}{|c|c|c|}
\hline \multicolumn{1}{|c|}{ Definition } & Symbol & TrFN \\
\hline \multirow{2}{*}{$\begin{array}{l}\text { Selling price for } \\
j \text {-th retailer }\end{array}$} & $\bar{C}_{P_{1}}$ & $(55,57,59,64)$ USD per ton \\
\cline { 2 - 3 } & $\bar{C}_{P_{2}}$ & $(52,56,59,62)$ USD per ton \\
\hline
\end{tabular}


Table 4. TrFN Price and transportation costs from supplier to retailer (USD per ton)

\begin{tabular}{|c|c|c|c|c|c|}
\hline \multicolumn{2}{|c|}{ Suppliers } & $S_{1}$ & $S_{2}$ & $S_{3}$ & $S_{4}$ \\
\hline \multirow{2}{*}{$\begin{array}{l}L=1, \\
\text { sea }\end{array}$} & $R_{1}$ & - & $(1900,1940,1980,2200)$ & $(2000,2100,2200,2300)$ & - \\
\hline & $R_{2}$ & - & $(1920,1940,1990,2100)$ & $(1900,2000,2100,2200)$ & - \\
\hline \multirow{2}{*}{$\begin{array}{l}L=2, \\
\text { truck }\end{array}$} & $R_{1}$ & $(230,290,320,390)$ & $(1450,1600,1650,1800)$ & - & $(1970,1990,2020,2100)$ \\
\hline & $R_{2}$ & $(210,295,310,400)$ & $(1550,1650,1700,1800)$ & - & $(1890,1990,2020,2200)$ \\
\hline \multirow{2}{*}{$\begin{array}{l}L=3, \\
\text { rail }\end{array}$} & $R_{1}$ & $(300,390,420,490)$ & $(1250,1400,1450,1600)$ & $(2040,2140,2200,2400)$ & $(950,990,1020,1100)$ \\
\hline & $R_{2}$ & $(310,395,410,500)$ & $(1350,1450,1500,1600)$ & $(2080,2180,2250,2490)$ & $(990,990,1020,1200)$ \\
\hline
\end{tabular}

Table 5. TrFN Transfer time of transported goods from supplier to retailer (per week)

\begin{tabular}{|l|c|c|c|c|c|}
\hline \multicolumn{2}{|c|}{ Suppliers } & $S_{1}$ & $S_{2}$ & $S_{3}$ & $S_{4}$ \\
\hline \multirow{2}{*}{$\begin{array}{l}L=1 \\
\text { sea }\end{array}$} & $R_{1}$ & - & $(3.5,4,4.5,5)$ & $(2.5,2.5,3,4)$ & - \\
\cline { 2 - 6 } & $R_{2}$ & - & $(3.5,4,4.5,5)$ & $(2.5,2.5,3,4)$ & - \\
\hline \multirow{2}{*}{$\begin{array}{l}L=2, \\
\text { truck }\end{array}$} & $R_{1}$ & $(2,3,4,5)$ & $(3,3.5,3.5,4)$ & - & $(1.5,2,2.5,2.5)$ \\
\cline { 2 - 6 } & $R_{2}$ & $(2.5,3.5,4,4.5)$ & $(3,3,4,4.5)$ & - & $(1.5,2,2,2.5)$ \\
\hline \multirow{2}{*}{$\begin{array}{l}L=3, \\
\text { rail }\end{array}$} & $R_{1}$ & $(2,3,4,5)$ & $(2.5,3,3.5,3.5)$ & $(2.5,2.5,3,4)$ & $(2,2.5,2.5,3)$ \\
\cline { 2 - 6 } & $R_{2}$ & $(2.5,3.5,4,4.5)$ & $(2.5,3,3,4)$ & $(2.5,2.5,3,4)$ & $(2,2.5,3,3.5)$ \\
\hline
\end{tabular}

On the basis of the above data, the binary multi objective fuzzy transportation model, Equation (32), is formulated as:

$$
\begin{aligned}
& \text { Min } Z_{1}= \\
& (1900,1940,1980,2200) \cdot x_{211}+ \\
& (2000,2100,2200,2300) \cdot x_{311}+ \\
& (1920,1940,1990,2100) \cdot x_{221}+ \\
& (1900,2000,2100,2200) \cdot x_{321}+ \\
& (230,290,320,390) \cdot x_{112}+ \\
& (210,295,310,400) \cdot x_{122}+ \\
& (1450,1600,1650,1800) \cdot x_{212}+ \\
& (1550,1650,1700,1800) \cdot x_{222}+ \\
& (1970,1990,2020,2100) \cdot x_{412}+ \\
& (1890,1990,2020,2200) \cdot x_{422}+ \\
& (300,390,420,490) \cdot x_{113}+ \\
& (310,395,410,500) \cdot x_{123}+ \\
& (1250,1400,1450,1600) \cdot x_{213}+ \\
& (1350,1450,1500,1600) \cdot x_{223}+ \\
& (2040,2140,2200,2400) \cdot x_{313}+ \\
& (2080,2180,2250,2490) \cdot x_{323}+ \\
& (950,990,1020,1100) \cdot x_{413}+ \\
& (990,990,1020,1200) \cdot x_{423} ; \\
& \text { Min } Z_{2}= \\
& (3.5,4,4.5,5) \cdot y_{211}+ \\
& (3.5,4,4.5,5) \cdot y_{221}+ \\
& (2.5,2.5,3,4) \cdot y_{311}+ \\
& (2.5,2.5,3,4) \cdot y_{321}+ \\
& (19
\end{aligned}
$$

$(2,3,4,5) \cdot y_{112}+$

$(2.5,3.5,4,4.5) \cdot y_{122}+$

$(3,3.5,3.5,4) \cdot y_{212}+$

$(3,3.5,3.5,4) \cdot y_{222}+$

$(1.5,2,2.5,2.5) \cdot y_{412}+$

$(1.5,2,2,2.5) \cdot y_{422}+$

$(2,3,4,5) \cdot y_{113}+$

$(2.5,3.5,4,4.5) \cdot y_{123}+$

$(2.5,3,3.5,3.5) \cdot y_{213}+$

$(2.5,3,3,4) \cdot y_{223}+$

$(2.5,2.5,3,4) \cdot y_{313}+$

$(2.5,2.5,3,4) \cdot y_{323}+$

$(2,2.5,2.5,3) \cdot y_{413}+$

$(2,2.5,3,3.5) \cdot y_{423}$

subject to

$$
\begin{aligned}
& x_{211}+x_{311}+x_{112}+x_{212}+ \\
& x_{412}+x_{113}+x_{213}+x_{313}+x_{413}=\bar{D}_{1} ; \\
& x_{221}+x_{321}+x_{122}+x_{222}+ \\
& x_{422}+x_{123}+x_{223}+x_{323}+x_{423}=\bar{D}_{2} ; \\
& x_{112}+x_{122}+x_{113}+x_{123} \leq 5000000 ; \\
& x_{211}+x_{221}+x_{212}+ \\
& x_{222}+x_{213}+x_{223} \leq 50000000 ; \\
& x_{311}+x_{321}+x_{313}+x_{323} \leq 50000000 \\
& x_{412}+x_{422}+x_{413}+x_{423} \leq 20000000 \\
& 15000 \cdot y_{211} \leq x_{211} \leq 25000000 \cdot y_{211} ; \\
& 15000 \cdot y_{221} \leq x_{221} \leq 25000000 \cdot y_{221} ;
\end{aligned}
$$




$$
\begin{aligned}
& 15000 \cdot y_{311} \leq x_{311} \leq 25000000 \cdot y_{311} ; \\
& 15000 \cdot y_{321} \leq x_{321} \leq 25000000 \cdot y_{321} \\
& 1500 \cdot y_{112} \leq x_{112} \leq 12000000 \cdot y_{112} ; \\
& 1500 \cdot y_{122} \leq x_{122} \leq 12000000 \cdot y_{122} ; \\
& 1500 \cdot y_{212} \leq x_{212} \leq 12000000 \cdot y_{212} ; \\
& 1500 \cdot y_{222} \leq x_{222} \leq 12000000 \cdot y_{222} ; \\
& 1500 \cdot y_{412} \leq x_{412} \leq 12000000 \cdot y_{412} ; \\
& 1500 \cdot y_{422} \leq x_{422} \leq 12000000 \cdot y_{422} ; \\
& 5000 \cdot y_{113} \leq x_{113} \leq 15000000 \cdot y_{113} ; \\
& 5000 \cdot y_{123} \leq x_{123} \leq 15000000 \cdot y_{123} ; \\
& 5000 \cdot y_{213} \leq x_{213} \leq 15000000 \cdot y_{213} ; \\
& 5000 \cdot y_{223} \leq x_{223} \leq 15000000 \cdot y_{223} ; \\
& 5000 \cdot y_{313} \leq x_{313} \leq 15000000 \cdot y_{313} ; \\
& 5000 \cdot y_{323} \leq x_{323} \leq 15000000 \cdot y_{323} ; \\
& 5000 \cdot y_{413} \leq x_{413} \leq 15000000 \cdot y_{413} ; \\
& 5000 \cdot y_{423} \leq x_{423} \leq 15000000 \cdot y_{423} ; \\
& \bar{D}_{1}=1.4 \cdot 10^{8} \cdot(55,57,59,64)^{-1.1} ; \\
& \bar{D}_{2}=1.2 \cdot 10^{8} \cdot(52,56,59,62)-1.3 \\
& y_{i j l}: 0 \text { or } 1 ; i=1,2 ; j=1,2 ; l=1,2,3 \\
& x_{211} \geq 0 ; i=1,2 ; j=1,2 ; l=1,2,3
\end{aligned}
$$

Two fuzzy constraint of this model are transformed into their non-fuzzy counterparts, using Equation (36):

$$
\begin{aligned}
& x_{211}+x_{311}+x_{112}+x_{212}+x_{412}+ \\
& x_{113}+x_{213}+x_{313}+x_{413}= \\
& 1.4 \cdot 10^{8} \cdot\left(\alpha \cdot\left(\frac{1}{55^{-1.1}}+\frac{1}{57^{-1.1}}\right)+\right. \\
& \left.(1-\alpha) \cdot\left(\frac{1}{59^{-1.1}}+\frac{1}{64^{-1.1}}\right)\right) ; \\
& x_{221}+x_{321}+x_{122}+x_{222}+x_{422}+ \\
& x_{123}+x_{223}+x_{323}+x_{423}= \\
& 1.2 \cdot 10^{8} \cdot\left(\alpha \cdot\left(\frac{1}{52^{-1.3}}+\frac{1}{56^{-1.3}}\right)+\right. \\
& \left.(1-\alpha) \cdot\left(\frac{1}{59^{-1.3}}+\frac{1}{62^{-1.3}}\right)\right) .
\end{aligned}
$$

Besides, the fuzzy objective functions are handled using their expected value, as shown in Equations (39) and (40). The ideal solutions can be found by letting $\alpha=$ 0 and solving the problems in Equations (37) and (38). The nadir-ideal solutions also obtained by changing the optimization direction in the abovementioned problems from "min" to "max". These values are obtained as $C^{-}=9241450000, C^{+}=1281361000, T^{-}=57.375$ and $T^{+}=4.125$. Now, the objective function of the problem in Equation (45) is formulated as:

$$
\operatorname{Max} \frac{9241450000-E V\left(Z_{1}\right)}{9241450000-1281361000}+\frac{57.375-E V\left(Z_{2}\right)}{57.375-4.125} \text {. }
$$

This problem is solved by letting $\alpha=0+0.1 \cdot k, k=$ $0,1, \ldots, 10$. The results are shown in the Table 6 .

Table 6. The satisfaction degree of the objective function

\begin{tabular}{|c|c|}
\hline$\alpha$ & Objective function \\
\hline 0 & 1.943662 \\
\hline 0.1 & 1.941704 \\
\hline 0.2 & 1.939747 \\
\hline 0.3 & 1.937789 \\
\hline 0.4 & 1.935832 \\
\hline 0.5 & 1.933874 \\
\hline 0.6 & 1.931917 \\
\hline 0.7 & 1.929959 \\
\hline 0.8 & 1.928002 \\
\hline 0.9 & 1.926044 \\
\hline 1 & 1.924087 \\
\hline
\end{tabular}

As it can be seen, increasing the value of $\alpha$, the obtained objective function (sum of satisfaction degree for both objectives) is decreased. This is because while increasing $\alpha$, the ambiguity of the problem decreases and the problem's feasible space is down-sized. Thus, decision maker(s) must make a trade-off between increasing certainty on one hand, and losing objective functions satisfaction degrees on the other hand. Considering this tradeoff, decision maker(s) in this case agreed on the satisfaction level of $60 \%$. On the level of $\alpha=0.6, y_{112}^{*}=y_{122}^{*}=1$, $x_{112}^{*}=3125214$ and $x_{122}^{*}=1271360$. This means that, all the demands of $R_{1}$ and $R_{2}$ are met by the domestic source. Also, the orders are transported using the second transportation mode, i.e. by truck.

\section{Conclusions}

TPs are used since the organization of human beings in to society, and today they have wide ranging applications such as scheduling, production, investment, plant location, inventory control, employment scheduling and many others. In this research optimizing inbound and outbound transportation costs of a multi echelon supply chain has been considered. To deal with uncertain time deliveries and pricing strategies adopted by different members of supply chain, fuzzy logic and specifically TrFNs are included. After designing a fuzzy binary multi objective model based upon structural assumptions, the solving approach is proposed and the model is employed on Iranian Steel Supply network to illustrate the potential and advantages of our scheduled model.

Integrating fuzzy logic and TrFNs for uncertain time and price quantities; besides, applying binary variables in conjunction with continuous variables to define transportation vessel type and order quantities; moreover, proposing a binary multi objective fuzzy transportation novel model and solving approach and finally, employing 
the proposed model in Iranian Steel supply network are the significant advantages of our research. The proposed model can be used for unlimited multi- echelon supply chains as we have performed in a supply chain network framework. Moreover prior researches optimized a single objective one stage TP, although a more complex multi stage TP has been optimized using the novel binary multi objective model. For future possible studies, considering more integrated supply networks, using stochastic demand functions and considering shipping line companies' policies as new constraint are notable.

\section{References}

Alam, T.; Rastogi, R. 2011. Transportation problem: extensions and methods - an overview, VSRD International Journal of Business and Management Research 1(2): 121-126.

Allahviranloo, T.; Firozja, M. A. 2007. Note on "Trapezoidal approximation of fuzzy numbers", Fuzzy Sets and Systems 158(7): 755-756. https://doi.org/10.1016/j.fss.2006.10.017

Amoozad Mahdiraji, H.; Razavi Hajiagha, S. H.; Hashemi, S. S.; Zavadskas, E. K. 2016. A grey multi-objective linear model to find critical path of a project by using time, cost, quality and risk parameters, E+M Ekonomie a Management 19(1): 49-61. https://doi.org/10.15240/tul/001/2016-1-004

Amoozad Mahdiraji, H.; Razavi Hajiagha, S. H.; Pourjam, R. 2011. A grey mathematical programming model to time-cost trade-offs in project management under uncertainty, in Proceedings of 2011 IEEE International Conference on Grey Systems and Intelligent Services, 15-18 September 2011, Nanjing, China, 698-704. https://doi.org/10.1109/GSIS.2011.6044123

Amoozad Mahdiraji, H.; Zavadskas, E.; Razavi Hajiagha, S. H. 2015. Game theoretic approach for coordinating unlimited multi echelon supply chains, Transformations in Business \& Economics 14(2): 133-151.

Bai, G.; Mao, J.; Lu, G. 2004. Grey transportation problem, Kybernetes 33(2): 219-224. https://doi.org/10.1108/03684920410514148

Ban, A. I.; Coroianu, L. 2014. Existence, uniqueness and continuity of trapezoidal approximations of fuzzy numbers under a general condition, Fuzzy Sets and Systems 257: 3-22. https://doi.org/10.1016/j.fss.2013.07.004

Basirzadeh, H. 2011. An approach for solving fuzzy transportation problem, Applied Mathematical Sciences 5(29-32): 1549-1566.

Chanas, S. 2001. On the interval approximation of a fuzzy number, Fuzzy Sets and Systems 122(2): 353-356. https://doi.org/10.1016/S0165-0114(00)00080-4

Chanas, S.; Kołodziejczyk, W.; Machaj, A. 1984. A fuzzy approach to the transportation problem, Fuzzy Sets and Systems 13(3): 211-221. https://doi.org/10.1016/0165-0114(84)90057-5

Chanas, S.; Kuchta, D. 1996. A concept of the optimal solution of the transportation problem with fuzzy cost coefficients, Fuzzy Sets and Systems 82(3): 299-305.

https://doi.org/10.1016/0165-0114(95)00278-2

Chen, S.-J.; Chen, S.-M. 2007. Fuzzy risk analysis based on the ranking of generalized trapezoidal fuzzy numbers, Applied Intelligence 26(1): 1-11.

https://doi.org/10.1007/s10489-006-0003-5

Chiang, J. 2005. The optimal solution of the transportation problem with fuzzy demand and fuzzy product, Journal of Information Science and Engineering 21(2): 439-451.
Delgado, M.; Vila, M. A.; Voxman, W. 1998. On a canonical representation of fuzzy numbers, Fuzzy Sets and Systems 93(1): 125-135. https://doi.org/10.1016/S0165-0114(96)00144-3

Deng, J. L. 1989. Introduction to Grey system theory, The Journal of Grey System 1(1): 1-24.

Dubois, D.; Prade, H. 1987. The mean value of a fuzzy number, Fuzzy Sets and Systems 24(3): 279-300. https://doi.org/10.1016/0165-0114(87)90028-5

Ebrahimnejad, A. 2014. A simplified new approach for solving fuzzy transportation problems with generalized trapezoidal fuzzy numbers, Applied Soft Computing 19: 171-176. https://doi.org/10.1016/j.asoc.2014.01.041

Esmaeili, M.; Aryanezhad, M.-B.; Zeephongsekul, P. 2009. A game theory approach in seller-buyer supply chain, European Journal of Operational Research 195(2): 442-448. https://doi.org/10.1016/j.ejor.2008.02.026

Gani, A. N.; Samuel, A. E.; Anuradha, D. 2011. Simplex type algorithm for solving fuzzy transportation problem, Tamsui Oxford Journal of Information and Mathematical Sciences 27(1): 89-98.

Grzegorzewski, P. 2008. New algorithms for trapezoidal approximation of fuzzy numbers preserving the expected interval, in Proceedings of IPMU'08, 22-27 June 2008, Torremolinos, Málaga, Spain, 117-123.

Grzegorzewski, P. 1998. Metrics and orders in space of fuzzy numbers, Fuzzy Sets and Systems 97(1): 83-94.

https://doi.org/10.1016/S0165-0114(96)00322-3

Güzel, N. 2010. Fuzzy transportation problem with the fuzzy amounts and the fuzzy costs, World Applied Sciences Journal 8(5): 543-549.

Heilpern, S. 1992. The expected value of a fuzzy number, Fuzzy Sets and Systems 47(1): 81-86. https://doi.org/10.1016/0165-0114(92)90062-9

Jia, P.; Amoozad Mahdiraji, H.; Govindan, K.; Meidutè, I. 2013. Leadership selection in an unlimited three-echelon supply chain, Journal of Business Economics and Management 14(3): 616-637. https://doi.org/10.3846/16111699.2012.761648

Jiménez, F.; Verdegay, J. L. 1999. Solving fuzzy solid transportation problems by an evolutionary algorithm based parametric approach, European Journal of Operational Research 117(3): 485-510. https://doi.org/10.1016/S0377-2217(98)00083-6

Jiménez, F.; Verdegay, J. L. 1998. Uncertain solid transportation problems, Fuzzy Sets and Systems 100(1-3): 45-57. https://doi.org/10.1016/S0165-0114(97)00164-4

Jin, S.; Lee, J.; Gen, M. 2011. Multi-product Two-stage Logistics Problem. Waseda University, Japan.

Kaur, A.; Kumar, A. 2012. A new approach for solving fuzzy transportation problems using generalized trapezoidal fuzzy numbers, Applied Soft Computing 12(3): 1201-1213. https://doi.org/10.1016/j.asoc.2011.10.014

Kocken, H. G.; Sivri, M. 2016. A simple parametric method to generate all optimal solutions of fuzzy solid transportation problem, Applied Mathematical Modelling 40(7-8): 46124624. https://doi.org/10.1016/j.apm.2015.10.053

Kumar, A.; Gupta, A. 2011. Methods for solving fuzzy assignment problems and fuzzy travelling salesman problems with different membership functions, Fuzzy Information and Engineering 3(1): 3-21. https://doi.org/10.1007/s12543-011-0062-0

Kumar, A.; Gupta, A.; Sharma, M. K. 2010. Solving fuzzy bicriteria fixed charge transportation problem using a new fuzzy algorithm International Journal of Applied Science and Engineering 8(1): 77-98. 
Kumar, B. R.; Murugesan, S. 2012. On fuzzy transportation problem using triangular fuzzy numbers with modified revised simplex method, International Journal of Engineering Science and Technology 4(1): 285-294.

Kundu, P.; Kar, S.; Maiti, M. 2015. Multi-item solid transportation problem with type-2 fuzzy parameters, Applied Soft Computing 31: 61-80. https://doi.org/10.1016/j.asoc.2015.02.007

Li, Q.-X.; Liu, S.; Wang, N.-A. 2014. Covered solution for a grey linear program based on a general formula for the inverse of a grey matrix, Grey Systems: Theory and Application 4(1): 72-94. https://doi.org/10.1108/GS-10-2013-0023

Lin, F.-T. 2009. Solving the transportation problem with fuzzy coefficients using genetic algorithms, in 2009 IEEE International Conference on Fuzzy Systems, 20-24 August 2009, Jeju Island, South Korea, 1468-1473.

https://doi.org/10.1109/FUZZY.2009.5277202

Liu, P.; Yang, L.; Wang, L.; Li, S. 2014. A solid transportation problem with type-2 fuzzy variables, Applied Soft Computing 24: 543-558. https://doi.org/10.1016/j.asoc.2014.08.005

Liu, S.; Lin, Y. 2006. Grey Information: Theory and Practical Applications. Springer. 508 p. https://doi.org/10.1007/1-84628-342-6

Mohanaselvi, S.; Ganesan, K. 2012. Fuzzy optimal solution to fuzzy transportation problem: a new approach, International Journal on Computer Science and Engineering 4(3): 367-386.

Pandian, P.; Natarajan, G. 2010. A new algorithm for finding a fuzzy optimal solution for fuzzy transportation problems, Applied Mathematical Sciences 4(2): 79-90.

Petraška, A.; Čižiūnienè, K.; Jarašūnienè, A.; Maruschak, P.; Prentkovskis, O. 2017. Algorithm for the assessment of heavyweight and oversize cargo transportation routes, Journal of Business Economics and Management 18(6): 1098-1114. https://doi.org/10.3846/16111699.2017.1334229

Poonam, S.; Abbas, S. H.; Gupta, V. K. 2012. Fuzzy transportation problem of triangular numbers with $\alpha$ - cut and ranking technique, IOSR Journal of Engineering 2(5): 1162-1164. https://doi.org/10.9790/3021-020511621164

Pramanik, S.; Jana, D. K.; Mondal, S. K.; Maiti, M. 2015. A fixedcharge transportation problem in two-stage supply chain network in Gaussian type-2 fuzzy environments, Information Sciences 325: 190-214. https://doi.org/10.1016/j.ins.2015.07.012

Purusotham, S.; Murthy, S. 2012. An exact algorithm for multi product bulk transportation problem, International Journal on Computer Science and Engineering 3(9): 3222-3236.

Razavi Hajiagha, S. H.; Mahdiraji, H. A.; Hashemi, S. S. 2014. A hybrid model of fuzzy goal programming and grey numbers in continuous project time, cost, and quality tradeoff, The International Journal of Advanced Manufacturing Technology 71(1-4): 117-126. https://doi.org/10.1007/s00170-013-5463-2

Rianthong, N.; Dumrongsiri, A. 2012. A mathematical model for optimal production, inventory and transportation planning with direct shipment, International Proceedings of Economics Development and Research 36: 23-27.

Ritha, W.; Vinotha, J. M. 2009. Multi-objective two stage fuzzy transportation problem, Journal of Physical Sciences 13: 107-120.

Samuel, A. E.; Venkatachalapathy, M. 2014. Improving IZPM for unbalanced fuzzy transportation problems, International Journal of Pure and Applied Mathematics 94(3): 419-424. https://doi.org/10.12732/ijpam.v94i3.9

Sharma, G.; Abbas, S. H.; Gupta, V. K. 2012. Solving transportation problem with the help of integer programming problem, IOSR Journal of Engineering 2(6): 1274-1277.

https://doi.org/10.9790/3021-026112741277
Stević, Ž; Pamučar, D.; Zavadskas, E. K.; Ćirović, G.; Prentkovskis, O. 2017. The selection of wagons for the internal transport of a logistics company: a novel approach based on rough BWM and rough SAW methods, Symmetry 9(11): 1-25. https://doi.org/10.3390/sym9110264

Wang, Y.-J. 2015. Ranking triangle and trapezoidal fuzzy numbers based on the relative preference relation, Applied Mathematical Modelling 39(2): 586-599. https://doi.org/10.1016/j.apm.2014.06.011

Yeh, C.-T. 2008. Trapezoidal and triangular approximations preserving the expected interval, Fuzzy Sets and Systems 159(11): 1345-1353. https://doi.org/10.1016/j.fss.2007.09.010

Zadeh, L. A. 1965. Fuzzy sets, Information and Control 8(3): 338-353. https://doi.org/10.1016/S0019-9958(65)90241-X

Zimmermann, H.-J. 1978. Fuzzy programming and linear programming with several objective functions, Fuzzy Sets and Systems 1(1): 45-55. https://doi.org/10.1016/0165-0114(78)90031-3 